\title{
A CONSULTA DE ENFERMAGEM NO CUIDADO À PESSOA COM CÂNCER: CONTEXTUALIZANDO UMA REALIDADE
}

\author{
Luciana Martins da Rosa ${ }^{1}$, Nen Nalú Alves das Mercês², Silvana Romagna Marcelino ${ }^{3}$, Vera Radünz ${ }^{4}$
}

\begin{abstract}
RESUMO: Este artigo é o resultado de uma prática assistencial que problematizou o desenvolvimento da consulta de enfermagem com dez enfermeiras da Central de Quimioterapia de uma Instituição de Saúde especializada no atendimento oncológico, no sul do Brasil. Os passos do Arco de Charles Maguerez nortearam a problematização e o método de pesquisa convergente-assistencial possibilitou, neste estudo, conciliar a pesquisa com o cuidado de enfermagem. As Teorias de Enfermagem: Relações Interpessoais de Hildegard Peplau e Autocuidado de Dorothéa Orem foram o referencial teórico. Esta aplicação demonstrou que a sistematização do cuidado de enfermagem requer a prática da educação permanente.

PALAVRAS-CHAVE: Cuidados de enfermagem; Consulta de enfermagem; Oncologia.
\end{abstract}

\section{NURSING CONSULTATION IN CARE DELIVERY TO THE ONCOLOGICAL PATIENT: CONTEXTUALIZINGA REALITY}

\begin{abstract}
This article is the result of a caring practice which outlined the development of nursing consultation with nurses from a Chemotherapy Central Unit, at a health Institution for oncological patients, in southern Brazil. The steps of Charles Maguerez's Arc supported problematization and the care-converging research method related research and nursing care in this study. The Nursing Theories: Hildegard Peplau's Interpersonal Relations and Dorothea Orem's Theory of Self Care were used as theoretical background. This application has demonstrated that systematization of nursing care requires a permanent education practice.

KEYWORDS: Nursing Care; Nursing Consultation; Oncology.

\section{LA CONSULTA DE ENFERMERÍA EN EL CUIDADO A LA PERSONA CON CÁNCER: CONTEXTUALIZANDO UNA REALIDAD}

RESUMEN: Este artículo es el resultado de una práctica asistencial que cuestionó el desarrollo de la consulta de enfermería con las enfermeras de la Central de Quimioterapia de una Institución de Salud especializada en la atención oncológica, en el Sur de Brasil. Los pasos del Arco de Charles Marguerez fueron utilizados para el desarrollo del problema. El modelo convergente y asistencial posibilitó, en este estudio, conciliar la investigación con el cuidado de enfermería. Las teorías de enfermería: Relaciones Interpesonales de Hildegard Peplau y Autocuidado de Dorothea Orem representaron el referencial teórico. Su aplicación demostró que la sistematización de los cuidados de enfermería necesita la práctica de la educación permanente.

PALABRAS CLAVE: Atención de enfermería; Consulta de enfermería; Oncología.

\footnotetext{
${ }^{1}$ Enfermeira do Centro de Pesquisas Oncológicas-CEPON/Secretaria de Estado da Saúde-SES/SC. Especialista em Projetos Assistênciais e Enfermagem Oncológica. Membro do grupo de pesquisa Cuidando e Confortando. Mestrando do Programa de Pós-Graduação em Enfermagem da Universidade Federal de Santa Catarina-UFSC.

${ }^{2}$ Enfermeira. Mestre em Assistência de Enfermagem pela UFSC. Doutoranda em Enfermagem/UFSC. Membro do Grupo de Pesquisa Cuidando e Confortando. Enfermeira do CEPON. Docente da Universidade do Vale do Itajaí.

${ }^{3}$ Enfermeira da SES/SC. Mestre em Assistência de Enfermagem pela UFSC. Doutorando em Enfermagem/UFSC. Docente da Universidade do Sul de Santa Catarina. Membro do grupo de pesquisa Educação e Enfermagem - EDEN.

${ }^{4}$ Enfermeira. Pós-Doutora pela University of Alberta/Canadá. Doutora em Enfermagem pela UFSC/Brasil. Docente dos Cursos de Graduação e Pós-Graduação do Departamento de Enfermagem da UFSC/Brasil. Membro e vice-líder do Grupo de Pesquisa Cuidando e Confortando da PEN/UFSC.
} 


\section{INTRODUÇÃO}

O ser e fazer da Enfermagem, ainda hoje é predominantemente atrelado à realização de procedimentos e à resolutividade de problemas imediatos. Isso pode decorrer da pouca valorização das ações de enfermagem e da dificuldade de visualização da abrangência da profissão pela sociedade e pelos próprios profissionais. O ser precisa se sobrepor ao fazer e isto acontece a partir da valorização, do reconhecimento e da realização pessoal e profissional. Estas necessidades estão relacionadas ao autoconhecimento, ao sentir-se profissional e à satisfação no desenvolvimento do trabalho, que depende de atitudes pessoais e institucionais ${ }^{(1)}$. A concretização do pensamento do ser e do fazer possibilita um cuidar mais humano. $\mathrm{O}$ homem que cuida é genuinamente humilde e está sempre disposto a aprender mais a respeito do cuidado e sobre si mesmo, incluindo o aprender por intermédio da pessoa cuidada ${ }^{(2)}$.

No cuidar existe uma relação entre o eu e o outro e, na Enfermagem, um destes momentos pode ser construído mediante a consulta de enfermagem. A consulta de enfermagem compreende: a etapa da coleta de dados, através do histórico de enfermagem e do exame físico; a etapa do planejamento da assistência, com o levantamento dos diagnósticos de enfermagem e prescrição; a etapa de execução do plano assistencial/ cuidados e implementação da assistência; e por último, as etapas de reavaliação e evolução.

A consulta de enfermagem consiste em uma atividade privativa do enfermeiro, que utiliza componentes do método científico e deve ser, obrigatoriamente, desenvolvida na assistência de enfermagem ${ }^{(3)}$. Podemos dizer que os enfermeiros realizam a consulta de enfermagem, principalmente nas áreas vinculadas à saúde coletiva e nos ambulatórios das diversas especialidades, mas observa-se a falta do registro adequado desta atividade. Há dificuldade na implantação e implementação do Processo de Enfermagem, decorrentes de diversas causas, desde a falta de um referencial teóricofilosófico do serviço, até a questões administrativas, dentre elas, o reduzido número de enfermeiros.

Na grande maioria das instituições não existe sistematização do cuidado de Enfermagem e o que se observa são ações de enfermagem sendo realizadas no imediatismo, na solução dos problemas e nas necessidades que vão surgindo. Ações que não são reavaliadas, que carecem de um plano de trabalho que contemple a diversidade e, ao mesmo, tempo garanta a prestação do cuidado, de acordo com a necessidade do contexto assistencial.

Enfatizamos que o "desenvolvimento da consulta de enfermagem ainda é designado como uma aplicabilidade do processo de enfermagem em nível ambulatorial"(4:41). Todavia, é uma atividade pertinente em âmbito ambulatorial, hospitalar, ocupacional, nas empresas e indústrias, bem como no ambiente domiciliar. Contudo, também existem serviços com consulta de enfermagem em escolas, asilos, penitenciárias entre outras instituições, em que se fazem necessárias as ações de prevenção das doenças, proteção e recuperação da saúde. Em alguns casos falta somente aproximar esta prática a um referencial teórico-filosófico, registrar o que é feito e compreender o que já está sendo executado.

O histórico da construção da consulta de enfermagem no Brasil se iniciou em 1925, por enfermeiras norte-americanas, com a elaboração de um manual utilizado nas entrevistas pós-clínicas desenvolvidas com pacientes com doenças sexualmente transmissíveis. No entanto, somente na década de 60 surgiu a denominação de consulta de enfermagem e as precursoras desta atividade são enfermeiras atuantes na saúde pública ${ }^{(4,5)}$. Neste sentido,

a consulta de enfermagem, antes de ser uma função inteiramente nova, na realidade consiste na reunião de várias tarefas em uma só atividade, sendo que estas atividades integram a prática de enfermagem há décadas ${ }^{(5: 84-5)}$.

No histórico da consulta de enfermagem na Oncologia do Brasil, temos o Instituto Nacional do Câncer (INCA), que realizou sua primeira consulta em 1993, a qual foi desenvolvida pela Enfermeira Luzia Medeiros. O Hospital do Câncer (HC) implantou a Sistematização daAssistência de Enfermagem (SAE) em 1994, o Centro de Pesquisas Oncológicas (CEPON) desenvolveu sua primeira consulta de enfermagem em 1997, como resultado da implantação da SAE e o Hospital Erasto Gaertner, em Curitiba -PR, iniciou a consulta de enfermagem em 2004, no atendimento das especialidades de gastroenterologia, ginecologia, dermatologia e otorrinolaringologia. Atualmente, esta instituição encontrase em processo de re-construção da SAE.

A instituição de saúde em que foi desenvolvido este estudo sobre a consulta de enfermagem adota o referencial teórico das Relações Interpessoais e do Autocuidado $^{(6)}$. A teoria das Relações Interpessoais, elaborada pela teórica Hildegard Peplau, propõe que a 
tomada de decisão terapêutica ocorra de forma interativa direta entre o paciente e a Enfermagem e de forma indireta com a família, sociedade e ambiente. A Enfermagem vivencia uma relação de afinidade com o paciente e trabalha a individualidade do ser, procurando encontrar significado nesta experiência ${ }^{(7)}$. $\mathrm{O}$ cuidado de Enfermagem proposto nesta teoria é humanístico e procura assistir as necessidades humanas, objetivando a superação do desconforto, da dúvida, da ansiedade e da insegurança. Além disto, prepara o paciente e a família para a independência, por intermédio de informações, esclarecimentos e controle de habilidades, procurando garantir o aprendizado necessário para o retorno ao lar e às atividades sociais ${ }^{(7)}$.

Esta teoria sugere fases seqüenciais das relações interpessoais: Orientação: fase de conhecimento e entrosamento entre paciente, enfermeiro e família, de reconhecimento de expectativa e de identificação de problemas. Identificação: fase da comunicação entre o profissional de enfermagem e o paciente, na qual ocorre a tomada de decisão e cada paciente pode apresentar uma maneira distinta de se relacionar, que pode ser por interdependência, independência ou dependência. Exploração: fase que o enfermeiro auxilia o conhecimento e a exploração dos caminhos disponíveis, ajudando na resolução do que foi identificado como problema. Solução: fase em que as metas planejadas foram alcançadas em decorrência da interação enfermeiro/paciente/família e de dissociação da relação terapêutica interpessoal.

A Teoria de Autocuidado, elaborada pela teórica Dorothéia Orem, é embasada na necessidade do ser humano quanto ao autocuidado e seu provimento. $\mathrm{O}$ indivíduo é capaz de autocuidar-se e quando não está preparado para tal ação submete-se ao cuidado de enfermagem, sendo que a Enfermagem deve oferecer condições para que o paciente possa aprender e sentir necessidade de autocuidado ${ }^{(7)}$.

Diante deste processo de aquisição de habilidades para o autocuidado, o enfermeiro auxilia e monitora esta exigência de forma contínua, mantendo a vida e a saúde, visando à recuperação de danos ou injúrias provenientes da doença, bem como auxiliar no enfrentamento dos efeitos da mesma ${ }^{(7)}$. Esta teoria se divide em: Teoria do Autocuidado: o indivíduo desenvolve o autocuidado. Teoria do Déficit do Autocuidado: diante dos desvios de saúde, surgem as limitações do autocuidado. A Enfermagem atua satisfazendo a demanda de autocuidado. Teoria de
Sistemas de Enfermagem: define o sistema de ações terapêuticas para o autocuidado, com o intuito de satisfazer as condições físicas, clínicas e emocionais do paciente.

Neste estudo utilizamos a abordagem de consulta de enfermagem como aquela que

funciona como um recurso para o diagnóstico de enfermagem ou identificação dos problemas de saúde do cliente e seu estudo em profundidade contribui para elaboração do plano de cuidados, para a atuação do enfermeiro e para melhoria da condição de saúde da população. Enfim, é a chave para atingir os objetivos da instituição e dos clientes. [...] enfermeiro e cliente buscam identificar os problemas e priorizar a resolutividade $[\ldots]^{(4: 41)}$.

A consulta de enfermagem surgiu na profissão como estratégia eficaz para detecção precoce de desvios de saúde e para acompanhar e dar seguimento às medidas instituídas ao bem-estar das pessoas envolvidas. Ela se diferencia dentre as várias maneiras de cuidar, pois possibilita a aproximação e estabelece uma relação interpessoal de ajuda concreta diante das variáveis culturais ${ }^{(4)}$.

Por causa das variáveis culturais, o desenvolvimento da consulta de enfermagem exige do enfermeiro a compreensão de cada ser, bem como do meio onde este vive. Quanto maior este conhecimento, maiores chances da consulta transformar os cuidados diários em cuidados imprescindíveis e condizentes com a realidade e com as necessidades individuais. Nesta perspectiva, a qualidade da interação entre a enfermeira e a pessoa com câncer, durante a consulta de enfermagem, é fator imprescindível para a eficácia da sistematização do cuidado de Enfermagem.

A enfermeira é o elo entre o cuidado e o conforto, assim a enfermeira oncológica precisa ser

[...] um ser humano possuidor de um "feeling" especial para com os outros e para com ela mesma, com competência na área de Enfermagem em Oncologia, que cuida de si mesmo e profissionalmente dos outros, que procura despertar nos outros e nela mesma a capacidade que o indivíduo tem para desempenhar os seus papéis e que desenvolve empatia ao interagir terapeuticamente ${ }^{(8:))}$.

Para finalizar os aspectos introdutórios, este estudo tem como objetivo problematizar o desenvolvimento da consulta de enfermagem com as enfermeiras da Central de Quimioterapia de uma Instituição de Saúde especializada no atendimento oncológico. 


\section{DESCRIÇÃO DO MÉTODO}

Este estudo adotou uma abordagem qualitativa a qual

articula a prática profissional com o conhecimento teórico, pois os seus resultados são canalizados progressivamente, durante o processo de pesquisa, para as situações práticas $^{(10: 26)}$.

Este método concilia a pesquisa com a assistência de enfermagem e observa-se a relação entre a situação atual, com a intencionalidade para buscar medidas resolutivas, gerando mudanças e inovações que repercutirão na situação social. O estudo qualitativo é capaz de agregar o significado e a intencionalidade como inerentes aos atos, às relações e às estruturas sociais ${ }^{(10)}$.

A pesquisa foi desenvolvida no período de outubro a dezembro de 2006, em uma Central de Quimioterapia Ambulatorial de uma Instituição de Saúde da Secretaria de Estado da Saúde de Santa Catarina, sendo referência no tratamento de Oncologia para o Estado. A Consulta de Enfermagem nesta unidade é realizada com todas as pessoas que iniciaram tratamento antiblástico ambulatorial e, durante a mesma, é fornecido um Manual de Orientações que contém informações sobre o tratamento antiblástico e os cuidados necessários durante e após os ciclos de quimioterapia.

Os sujeitos deste estudo foram dez enfermeiras que trabalham na Central de Quimioterapia da respectiva instituição e que concordaram em participar do estudo. O desenvolvimento da pesquisa ocorreu em cinco encontros, de aproximadamente uma hora, agendados previamente com as enfermeiras.

Para garantir o anonimato, cada enfermeira escolheu um codinome, dentre o nome de várias pedras preciosas. Obedecendo a legislação ${ }^{(11)}$ em vigor, o Comitê de Ética em Pesquisa da Instituição aprovou, pelo parecer 034/2006, o desenvolvimento desta pesquisa que foi iniciada após a apresentação da proposta do estudo e esclarecimentos necessários às enfermeiras, bem como com a assinatura do Termo de Consentimento Livre e Esclarecido.

Para alcançar o objetivo proposto, utilizou-se o método problematizador, mediante as etapas do Arco de Charles Maguerez, apresentado por Bordenave e Pereira ${ }^{(12)}$, composto dos passos: observação da realidade - definição dos pontos-chave - teorização e análise - hipótese de solução - aplicação à realidade. Portanto, o ponto de partida e de chegada é a realidade.

A problematização ocorre com a exposição dos envolvidos com o problema e somente acontece quando se observa a realidade, ou seja, a síncrese do assunto/problema. Há a identificação das variáveis ou pontos-chave do problema, aqueles que, se modificados, podem resultar na solução dos problemas. Com a teorização, há a discussão sobre o problema, que envolve estudos e pesquisa sobre o assunto em questão, pois a ciência pode fornecer os esclarecimentos necessários sobre o tema, facilitando a análise, propiciando as hipóteses de solução do problema e sua aplicação à realidade ${ }^{(12)}$.

\section{RESULTADO}

O cuidado é a base da Enfermagem e a consulta de enfermagem é uma ação integrante deste cuidado. Estamos cientes de que não desvelamos todas as faces da consulta de enfermagem à pessoa com câncer. No entanto, este estudo apresentou alguns pontos, os nós de uma prática de enfermeiras de um serviço de Oncologia.

Aplicando a problematização com o Arco de Charles Maguerez e tendo como realidade a consulta de enfermagem em oncologia, os seguintes pontoschave foram levantados:1) Formulário do histórico de enfermagem sem praticidade e favorecendo anotações similares às dos médicos; 2) Enfermeiras realizando as orientações de enfermagem de forma diversa e sem padronização científica; 3) Dificuldade de interação de algumas enfermeiras com a pessoa com câncer durante a consulta de enfermagem; 4) Desenvolvimento de consultas de enfermagem no período da tarde, na área física da Central de Quimioterapia; 5) Ausência de um manual de orientação para o desenvolvimento da consulta de enfermagem; 6) Ausência de atividades de educação permanente para a manutenção da SAE; 7) Identificação das necessidades ao invés do Diagnóstico de Enfermagem.

A partir da teorização e análise de cada pontochave, o grupo de enfermeiras assistenciais e a enfermeira facilitadora propuseram as hipóteses de solução que culminaram em: 1) Elaboração do Manual: "A Consulta de Enfermagem ao Paciente em Tratamento Antineoplásico: manual de orientação ao enfermeiro". Este manual foi elaborado com orientações caracterizadas pelas enfermeiras como informações gerais para todas as pessoas em tratamento antiblástico, independentemente do esquema de tratamento adotado pelo oncologista e com informações específicas para cada droga antiblástica utilizada na instituição, contendo também um quadro comparativo dos nomes 
farmacológicos, comerciais e siglas de cada medicamento. 2) Elaboração de um novo formulário para registro do Histórico de Enfermagem e aplicabilidade de 40 testes-piloto do formulário proposto. 3) Comprometimento das enfermeiras do período vespertino no desenvolvimento da consulta de enfermagem no consultório de enfermagem e não na Central de Quimioterapia, por dificultar a interação entre enfermeira e usuário. 4) Comprometimento das enfermeiras em manter estudos sobre a SAE, favorecendo assim a problematização constante e o envolvimento continuado com as proposições das teorias norteadoras da SAE e, conseqüentemente, contribuindo na qualificação da consulta de enfermagem e todas as ações subseqüentes a esta prática. 5) Identificação da necessidade de aprofundamento dos estudos sobre diagnóstico de enfermagem, sendo sugerido pela equipe a introdução da Associação Diagnóstica das Enfermeiras Norte-Americanas - North American Nursing Diagnosis Association (NANDA). E, para garantir a padronização dos diagnósticos entre as Unidades Institucionais, a equipe encaminhou os resultados obtidos à Gerência de Assistência e ao mesmo tempo reivindicou a manutenção da SAE em todas as Unidades da Instituição.

No último encontro foi aplicado um instrumento com seis questões abertas, com a finalidade de avaliar a pesquisa desenvolvida relativa à sistematização dos cuidados e da consulta de enfermagem.

Em relação ao questionamento de como as enfermeiras percebiam o desenvolvimento da consulta de enfermagem no seu Serviço, responderam.

Podemos avaliar o paciente como um todo, realizar um trabalho científico, minimizar efeitos colaterais com as orientações e comprovar trabalho realizado (Esmeralda).

É importante, para conhecermos o paciente e seu convivio familiar, sendo que neste primeiro momento ou acolhimento conseguimos identificar várias situações como medos, angústias, falta de conhecimento e outras (Ametista).

Os relatos anteriores evidenciam que as enfermeiras acreditam na importância da prática da consulta de enfermagem. Relataram que conhecer o outro possibilita o planejamento das ações de Enfermagem de forma científica e individualizada.

A consulta de enfermagem faz parte da amplitude imensa do cuidar, e "[...] o ser humano faz parte da natureza e se integra a ela para existir, resistir e interpretar a vida. $\mathrm{O}$ cuidado faz parte da Enfermagem e dá movimento às ações pela vida"(15).

Este resultado é confirmado, pois a problematização discutida durante todo o estudo enseja o aprendizado de pensar com uma visão global que observa a realidade e que leva a elaboração de estratégias para uma melhoria ${ }^{(12)}$.

Em relação ao questionamento da problematização da consulta de enfermagem para o desenvolvimento desta prática, as enfermeiras relataram:

Acredito que as conversas com o grupo nos fizeram buscar mais cientificidade, homogeneizar condutas, orientações no sentido de tornar o serviço mais comprometido e confiável (Diamante).

[...] no transcorrer do trabalho, às vezes, tornamonos mecanicistas e esta parada para avaliação é extremamente importante para mudarmos paradigmas (Pérola).

Os depoimentos confirmam a relevância deste estudo e registra a contribuição para as enfermeiras extrapolarem o fazer, despertando-as para se preocuparem com $o$ ser.

Em relação à indagação de conhecimento das Teorias: Processo Interpessoal, de Hildegard Peplau e Autocuidado, de Dorothéa Orem, bem como dos passos dessa teoria, das sete enfermeiras que responderam ao questionamento, duas disseram que não conheciam as teorias. Assim, concluímos que existe a necessidade de um estudo aprofundado das teorias na capacitação dos profissionais quando lotados no Serviço de Enfermagem da Instituição em foco, além de estudos periódicos para discussão dos conceitos norteadores.

Quanto ao questionamento se as enfermeiras utilizavam os passos sugeridos pelas teorias do estudo, as enfermeiras responderam:

Sim, porque os pacientes que são atendidos precisam praticar o autocuidado (Turquesa).

Nem sempre. Algumas vezes existe falta de estudo e esquecimento das teorias (Esmeralda).

Participei da implantação, assim os passos já estão introjetados (Pérola).

Pelos relatos acima, concluímos que existe a 
necessidade para as enfermeiras de uma maior compreensão do que consiste em utilizar e seguir uma sistematização da assistência de enfermagem, bem como a importância desta prática.

A seguir, fizemos o seguinte questionamento, considerando que a sistematização da assistência de enfermagem neste serviço foi implantada em 1997, e assim já possui quase dez anos de prática: "você considera necessário fazer alguma modificação?"

Acredito que as modificações já estão sendo encaminhadas através do desenvolvimento da problematização da consulta de enfermagem proposta por esta prática asssistencial (Pérola).

Há necessidade de aumentar os registros das ações de enfermagem, pois muitos ainda não são realizados (Água Marinha).

Existe a importância da padronização das ações $e$ dos registros das enfermeiras (Jade).

Também é necessário o estudo para aprimoramento das ações (Ametista).

Diante das respostas anteriores, observa-se que apesar da sistematização dos cuidados de enfermagem existir há nove anos, faz-se necessária a articulação e o envolvimento do grupo na solução dos problemas identificados nesta pesquisa, pois os mesmos vão além dos pontos-chave discutidos no início desta prática.

Acreditamos que a adoção da educação permanente na sistematização da assistência de enfermagem, partindo da situação problema, pode levar à superação, à mudança e à transformação da realidade em uma situação diferente e desejada ${ }^{(13)}$.

Para Soffiatti ${ }^{(14)}$, em relação à consulta de enfermagem em ambulatório de quimioterapia, a educação permanente do enfermeiro e da pessoa com câncer favorece o sucesso do tratamento e do cuidado de enfermagem. Estas considerações reforçam o resultado deste estudo e demonstram que apesar de ser uma conclusão lógica, ainda fazem-se necessários estudos e discussões para manutenção permanente do lógico.

Por último, indagamos aos participantes a respeito de como haviam percebido o novo modelo (teste-piloto) de formulário para desenvolvimento do histórico de enfermagem. Exemplificamos com algumas respostas.

Achei bom, pois foi construído por todos nós. Sendo assim, acho que o resultado será melhor ainda (Jade).

Mais prático, mais rápido e agradável de realizar (Esmeralda).

Destacamos somente duas respostas por considerarmos que as mesmas representaram a opinião de todas e ficou evidente que o novo instrumento, na opinião dos participantes, está melhor do que o adotado atualmente. Entretanto, a resposta de Jade reforça que quando existe o envolvimento de todos, permanentemente, os resultados tendem a ser mais eficazes. Desta forma, concluímos que não podemos iniciar a sistematização a partir do zero ou a cada inclusão de novo profissional, mas é possível seguir um movimento de transformação da realidade da prática profissional, através de uma observação crítica e construtiva com o envolvimento de todos os pares. A análise como processo é necessária para o fazer crítico e atender os critérios de cientificidade das ações. Para tanto, é necessário abrir espaços que possibilitem a reflexão acerca da realidade.

\section{CONSIDERAÇÕES FINAIS}

A problematização da consulta de enfermagem oportunizou as enfermeiras apontarem alternativas, a partir de suas experiências, favorecendo a construção de uma prática mais criativa, humana e reflexiva, levando-as a reavaliar o fazer na Enfermagem Oncológica. Além disto, o modelo convergente-assistencial possibilitou implementar as estratégias de acordo com as diretrizes do Programa de Qualidade existentes na Instituição de Saúde.

Avaliando os resultados obtidos, podemos afirmar que a SAE sustenta a prática de enfermagem apresentada, no entanto, é necessária a prática de educação permanente para fortalecer os métodos e conceitos estabelecidos pelas teorias norteadoras.

As mudanças propostas aproximaram a equipe da reavaliação dos conceitos científicos e de uma prática mais harmônica, procurando sistematizar as abordagens entre as diversas enfermeiras. Evidenciamos a atitude e o interesse profissional de cada enfermeira na participação das atividades desenvolvidas, na construção do manual de orientações e na adequação do formulário para registro do histórico de enfermagem, o que demonstrou o comprometimento do grupo em manter o espírito de união e o reconhecimento de que a educação permanente é uma necessidade. Um dos pontos fortes foi que a SAE não trata somente de um 
registro e sim de uma ação transformadora do pensar e do fazer da Enfermagem. Somente desta forma os cuidados de Enfermagem integrarão os itinerários de cuidados das pessoas com câncer.

Por outro lado, observou-se a importância do papel da enfermeira como educadora e implementadora da melhoria da qualidade dos serviços prestados. Esta observação concretizou-se na avaliação das enfermeiras quanto ao empenho na qualificação da consulta de enfermagem e na decisão de continuidade da educação permanente para o desenvolvimento da SAE.

O referencial teórico e metodológico favoreceu o desenvolvimento da problematização, pois as Teorias de Peplau e Orem sugerem passos que se aproximam aos passos do Arco de Charles Maguerez. Ainda, a abordagem de Trentini e Paim com a Pesquisa Convergente-Assistencial, contribuiu para aquisição de um salto qualitativo, porque parte da realidade e para ela retornar agregando valores.

Concluímos que este estudo contribuiu com $o$ ser e o fazer da Enfermagem e com o cuidado prestado à pessoa com câncer, além de problematizar o desenvolvimento da consulta de enfermagem com as enfermeiras da Central de Quimioterapia em uma unidade especializada no atendimento oncológico o que foi um desafio gratificante.

\section{REFERÊNCIAS}

1 Lentz RA, Costenaro RGS, Gonçalves LHT, Nassar SM. O profissional de enfermagem e a qualidade de vida: uma abordagem fundamentada nas dimensões propostas por Flanagan. Rev Latino-am Enferm. 2000 Ago;8(4):7-14.

2 Mayeroff R. On caring. New York: Harper \& Row; 1971.

3 Conselho Federal de Enfermagem (COFEN). Resolução $\mathrm{n}^{\circ} 159 / 1993$. Dispõe sobre a consulta de enfermagem. [acesso em 2006 Out 7]. Disponível em: www.portalcofen.gov.br.

4 Zagonel IPS. Consulta de enfermagem: um modelo de metodologia para o cuidado. In: Westphalen MEA, Carraro TE, organizadores. Metodologias para a assistência de enfermagem: teorizações, modelos e subsídios para a prática. Goiânia: AB; 2001. p. 41-56.

5 Castro IB. Estudo exploratório sobre a consulta de enfermagem. Rev Bras Enferm. 1975;4(28):76-94.

6 Avila LMR. Uma abordagem interacionista e de autocuidado para sistematização da assistência de enfermagem do CEPON. [monografia]. Florianópolis (SC): Universidade Federal de Santa Catarina;1997.
7 George JB. Teorias de enfermagem: os fundamentos para a prática profissional. Porto Alegre: Artes Médicas; 1993.

8 Radünz V. Cuidando e se cuidando: fortalecendo o self do cliente oncológica e o self da enfermeira. $2^{\mathrm{a}}$ ed. Goiânia: AB; 1999.

9 Trentini M, Paim L. Pesquisa convergente assistencial: um desenho que une o fazer e o pensar na prática assistencial em saúde-enfermagem. $2^{\mathrm{a}}$ ed. Florianópolis: Insular; 2004.

10 Minayo MCS. O desafio do conhecimento: pesquisa qualitativa em saúde. $6^{\mathrm{a}}$ ed. São Paulo:Hucitec-Abrasco; 1999.

11 Ministério da Saúde (BR). Conselho Nacional de Saúde. Diretrizes e normas regulamentadoras de pesquisa envolvendo seres humanos. Resolução $n^{\circ} 196$, de 10 de outubro de 1996. Brasília; 1996.

12 Bordenave JD, Pereira AMP. Estratégias de ensinoaprendizagem. 22a ed. Petrópolis: Editora Vozes; 2001.

13 Ricaldoni CAC, Sena RR. Educação permanente: uma ferramenta para pensar e agir no trabalho de enfermagem. Rev Latino-am Enferm. 2006 Nov/Dez; 14(6):837-42.

14 Soffiatti NRT. Consulta de enfermagem em ambulatório de quimioterapia: ênfase nas ações educativas. Cogitare Enferm. 2000 Jan/Jun;5(n ${ }^{\circ}$ esp):69-72.

15 Marcelino SR. Fundamentando a prática de enferma gem no referencial do cuidar/cuidado e ensinar/ aprender. In: Mercês NNA, Marcelino SR. Enfermagem oncológica: a representação social do câncer e o cuidado paliativo no domicílio. Blumenau: Nova Letra; 2004. p. 208-25. 